\title{
Integrating solids and gases for attosecond pulse generation
}

\author{
T. J. Hammond ${ }^{1,2 \star}$, Sylvain Monchocé1, Chunmei Zhang', Giulio Vampa ${ }^{1,3}$, Dennis Klug ${ }^{4}$, \\ A. Yu. Naumov ${ }^{4}$, D. M. Villeneuve ${ }^{4}$ and P. B. Corkum ${ }^{1,4}$
}

Control of the field of few-cycle optical pulses has had an enormous impact on attosecond science. Subcycle pulses open the potential for non-adiabatic phase matching while concentrating the electric field so it can be used most efficiently. However, subcycle field transients have been difficult to generate. We exploit the perturbative response of a sub-100 $\mu \mathrm{m}$ thick monocrystalline quartz plate irradiated by an intense few-cycle $1.8 \mu \mathrm{m}$ pulse, which creates a phase-controlled supercontinuum spectrum. Within the quartz, the pulse becomes space-time coupled as it generates a parallel second harmonic. Vacuum propagation naturally leads to a subcycle electric-field transient whose envelope is sculpted by the carrier envelope phase of the incident radiation. We show that a second medium (either gas or solid) can generate isolated attosecond pulses in the extreme ultraviolet region. With no optical elements between the components, the process is scalable to very high energy pulses and allows the use of diverse media.

Sister ynthesized field transients that are subcycle in duration have important potential for attosecond technology and for coherent control $^{1-7}$. However, to synthesize field transients is challenging because multiple dispersive elements are needed to compensate for the large chirp and large bandwidth that the technique requires ${ }^{8-14}$. We solved this challenge by operating in the anomalous dispersion region of crystalline X-cut quartz and by using high-intensity light. Combined, these choices enable us to use sub- $100 \mu \mathrm{m}$ thick samples that have minimum dispersion ${ }^{15,16}$. Passing through the sample, our $1.8 \mu \mathrm{m}$ beam is spectrally broadened, and within one coherence length approximately $2 \%$ of the beam energy is converted to broadband second harmonic radiation parallel to the fundamental field ${ }^{17}$. The emerging beam is an intense, reshaped version of the driver-compressed in space and time for attosecond science.

We use a second medium, placed approximately half a Rayleigh range away, to produce an intense, tunable and isolated attosecond pulse. Here we report experimental results obtained when the second medium is xenon, krypton, argon or a crystalline X-cut quartz sample, but the method is general and can be scaled in energy by the beam size. By scanning the carrier envelope phase (CEP) of the fundamental field, we tune the photon energy of the attosecond pulse. We also measure the spectral phase of the attosecond pulse generated in quartz and find that it has a qualitatively different behaviour from that of xenon ${ }^{18}$.

\section{Spatiotemporal compression}

Figure 1a illustrates the experimental set-up, and Fig. 1b-e shows simulations of the synthesized transient field. Quartz has a high optical-damage threshold because of its large bandgap $(9 \mathrm{eV})$, which allows us to use a two-cycle $\lambda=1.8 \mu \mathrm{m}$ beam with intensities up to $4 \times 10^{13} \mathrm{~W} \mathrm{~cm}^{-2}$ without multishot damage. Although crystalline quartz has a relatively low second-order susceptibility $\left(d_{\text {eff }}=0.3 \mathrm{pm}\right.$ $\mathrm{V}^{-1}$ ), at these intensities we convert a few percent of the fundamental into its second harmonic. Although this may seem small, the electric field is $\sim 15 \%$ of the fundamental.
To model the few-cycle laser pulse that propagates through quartz, we use the forward Maxwell equation ${ }^{19}$. We assume that the $200 \mu \mathrm{m}$ spot size remains constant through the thin $(80 \pm 10 \mu \mathrm{m})$ quartz. The initial simulated beam (Fig. 1b) has a Gaussian electric-field distribution in both time and space, and is at normal incidence to the quartz; the polarization is perpendicular to the $c$-axis direction of the crystal. The strong field generates new spectral components through self-phase modulation and second-harmonic generation, and the anomalous dispersion of the quartz compresses the pulse in time. Many of the newly created spectral components are near the region of zero dispersion (at $\lambda=1.28 \mu \mathrm{m}$ ) and remain in phase with the driving field over the thickness of the crystal. Simultaneously, the intensity-dependent nonlinear index of refraction delays the on-axis, high-intensity spatial portion of the beam, as shown in Fig. 1c. At the output face of the quartz, this distorted wavefront is space-time coupled ${ }^{20,21}$.

Once in the vacuum, we use a Henkel transform to model the propagation. With our parameters, the space-time coupled field reaches a maximum intensity approximately $2 \mathrm{~cm}$ from the quartz, where the pulse is also compressed in space. This region of maximum intensity depends on the beam waist, peak intensity, quartz thickness and CEP. At the new 'focus' (black in Fig. 1e), the main half-cycle has a higher contrast than immediately after the quartz plate (red), and the intensity is four times higher than the initial pulse (blue). The green dotted line shows the square of the electric field within the envelope situated at the region of maximum intensity. Depending on the distance from the quartz plate and the CEP, the full-width at half-maximum (FWHM) field transient can be as short as a half-cycle.

We measured the field evolution through the focus via the petahertz optical oscilloscope technique ${ }^{22}$, as shown in Fig. 2 (further details of this measurement are given in Supplementary Information). The columns are the position of the gas-jet measurement position, for $z=-3 \mathrm{~mm}$ (left column), $z=-1 \mathrm{~mm}$ (middle column) and $z=0 \mathrm{~mm}$ at the focus (right column). Figure $2 \mathrm{a}-\mathrm{c}$ shows the recorded petahertz optical oscilloscope spectrograms from the measurement of the extreme ultraviolet (XUV) deflection 

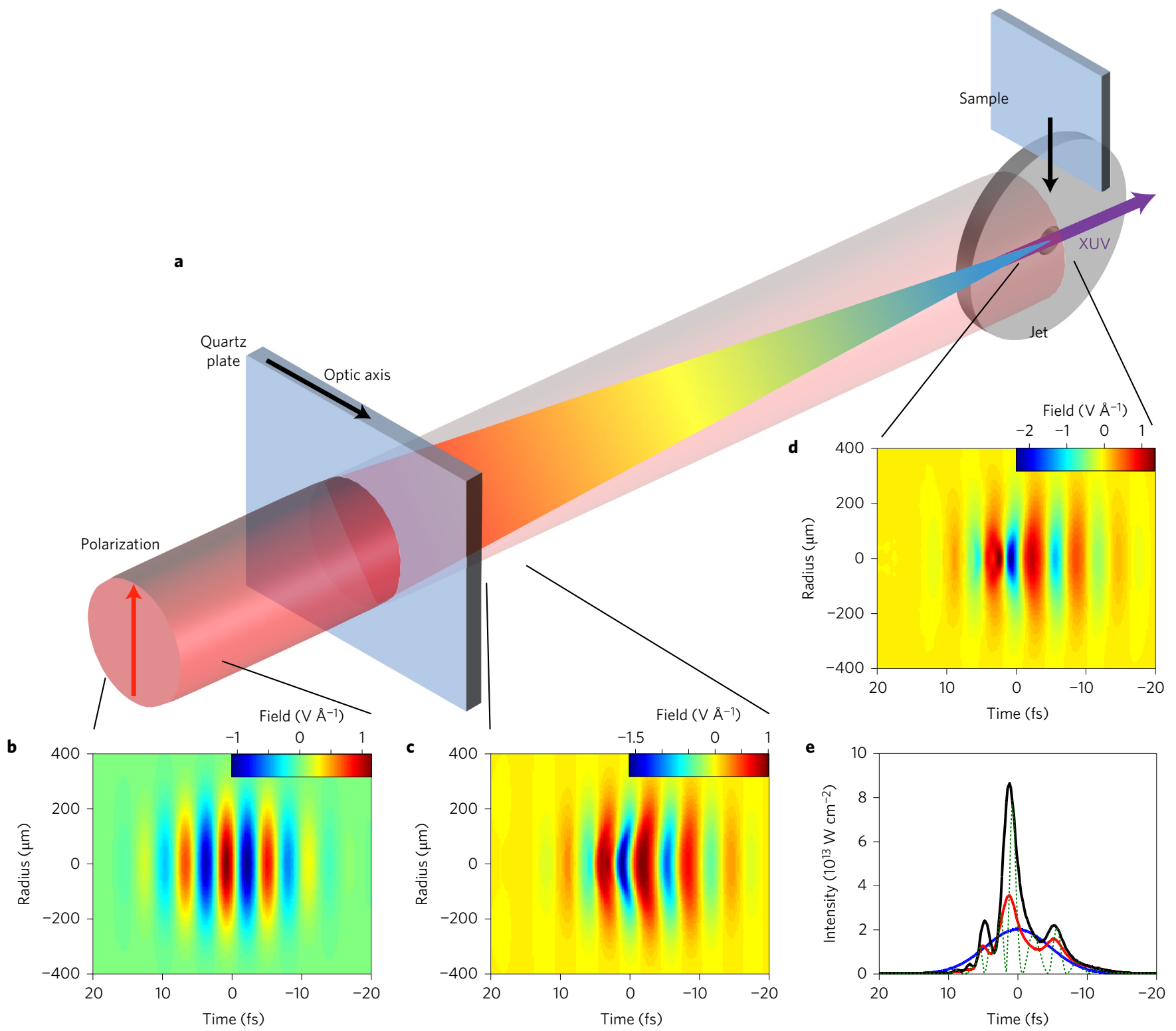

Figure 1 | Pulse compression via propagation through quartz and vacuum. a-e, Experimental set-up of half-cycle pulse generation and interaction with a gas jet or solid sample (a), and simulation of pulse propagation (b-e). b. A collimated, two-cycle pulse centred at $1.8 \mu \mathrm{m}$ with a peak intensity of $2 \times 10^{13} \mathrm{~W} \mathrm{~cm}^{-2}$ and a radius of $200 \mu \mathrm{m}$ is incident on $80 \mu \mathrm{m}$ of quartz and is polarized perpendicular to the $c$ axis of quartz. c, After propagation through the quartz plate, the nonlinear index of refraction radial dependence modifies the wavefront. $\mathbf{d}$, In vacuum, the pulse undergoes spatial and temporal compression and its peak intensity increases by $20 \mathrm{~mm}$ from the quartz, now optimized to generate an isolated attosecond pulse in gas (jet) or solid (sample). e, The intensity envelope of the initial pulse (blue), after quartz (red) and propagated $20 \mathrm{~mm}$ (black) shows the spatiotemporal compression; the resulting square of the field is shown by the green dots. The compressed pulse-envelope duration is $2.4 \mathrm{fs}$, whereas the half-cycle transient duration is $1.2 \mathrm{fs}$.

caused by the signal field as a function of delay. We fit the maximum of the spectrogram (white line) to obtain the signal $\sigma=\mathrm{d} E / \mathrm{d} t$, the derivative of the electric field. We filtered the signal from 0.4 to $4 \mu \mathrm{m}$ and integrated it to obtain the electric field (Fig. $2 \mathrm{~d}-\mathrm{f}$ ). The field evolution through the focus is more apparent when we square the measured field (Fig. $2 \mathrm{~g}-\mathrm{i}$ ). The measured field far before the focus, at $z=-3 \mathrm{~mm}$, shows a 1.5 -cycle pulse. As we approach the focus, at $z=-1 \mathrm{~mm}$, the pulse evolves into a halfcycle pulse, although with a contrast of $2: 1$. At the focus, the halfcycle transient improves the contrast to $2.5: 1$, where the FWHM pulse duration is $1.3 \mathrm{fs}$. This field evolution through the focus is expected because of the spatiotemporal coupling of the pulse, and we find that the pulse duration increases after the focus in a similar manner to before the focus.

\section{Octave-tunable isolated attosecond pulses}

We use these half-cycle transient fields for strong-field experiments. In the three-step model of high-harmonic generation ${ }^{23}$, the field separates a valence electron from an atom or molecule in one half-cycle and drives recombination in the second half-cycle, which releases the energy as a photon. With this half-cycle pulse, we can alter the ionization rate and the recollision energy by tuning the CEP. Figure 3 shows the high harmonic spectra for xenon as a function of the CEP-the colour bars represent amplitudes of XUV radiation relative to the few-cycle case. The left column is the calculated field for two different CEP values, the middle column is the calculated XUV spectrum as a function of CEP and the right column is the experiment. For the two-cycle pulse, Fig. $3 a-c$, we see a discrete harmonics spectrum, especially 


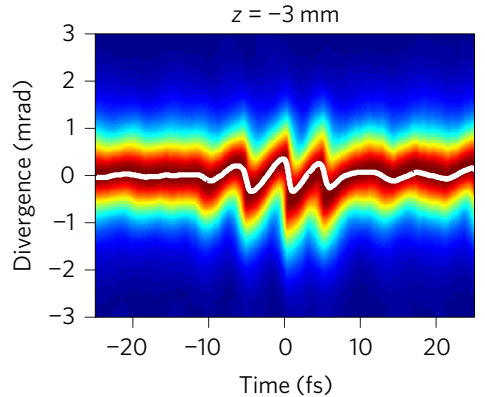

d

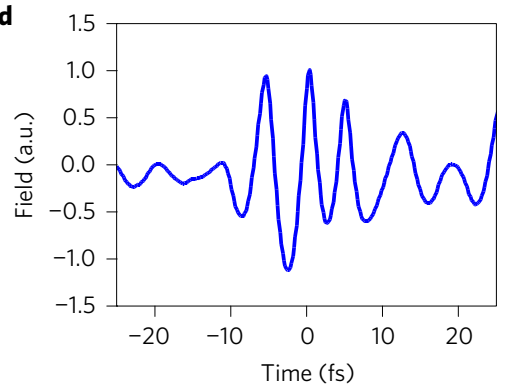

g

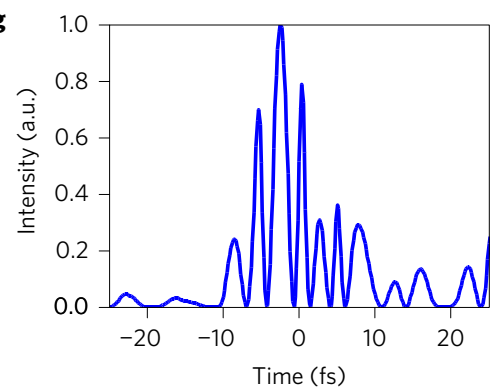

b

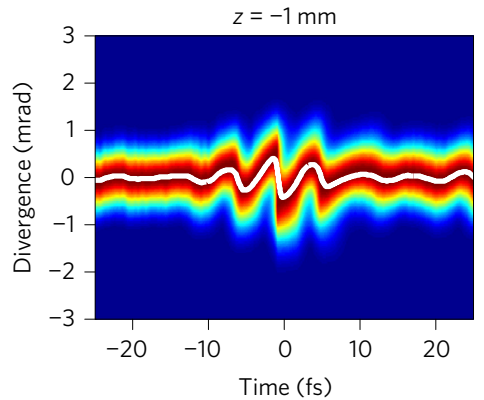

e

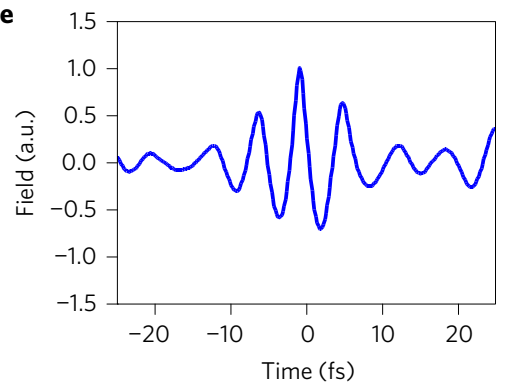

h

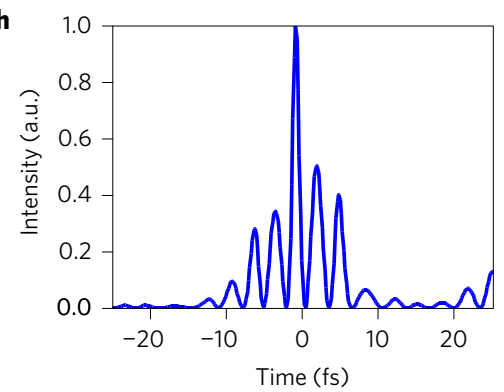

c

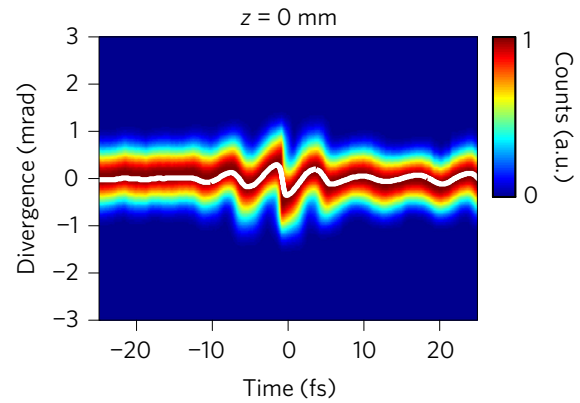

f

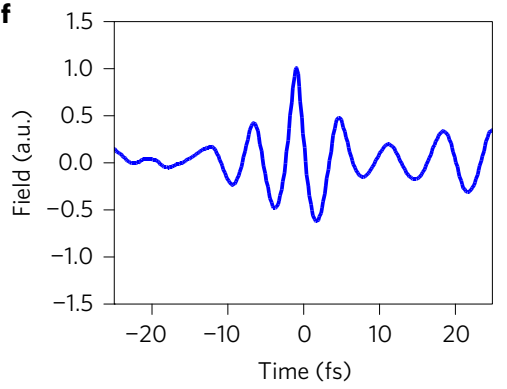

i

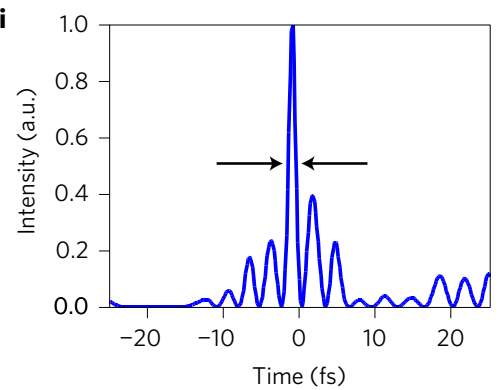

Figure 2 | Petahertz optical oscilloscope measurement of the electric field through the focus. a-c, Recorded spectrograms; the white lines are the fits. $\mathbf{d}-\mathbf{f}$, The resulting electric field. $\mathbf{g}-\mathbf{i}$, Square of the field. $\mathbf{a}$, Before the focus, at $z=-3 \mathrm{~mm}$, the field is a 1.5 cycle pulse. $\mathbf{b}$, Near the focus, at $z=-1 \mathrm{~mm}$, we measure a half-cycle pulse with a contrast of 2:1. c, At the focus, $z=0 \mathrm{~mm}$, the spatial-temporal coupling leads to a half-cycle field transient with a contrast of 2.5:1; the distance between the arrows indicates that the FWHM $\Delta t=1.3$ fs. a.u., arbitrary units.

in the low photon energy portion of the spectrum. The maximum photon energy, which is proportional to the change in the square of the peak field strength, changes by only a few electronvolts per $2 \pi$ change of the CEP. By introducing the quartz plate with the polarization of the $1.8 \mu \mathrm{m}$ beam parallel to the quartz $c$-axis direction (Fig. $3 \mathrm{~d}-\mathrm{f}$ ), we see a strong half-cycle cutoff that spans from 20 to $35 \mathrm{eV}$. The space-time coupling introduced by the quartz increases the driving field intensity and leads to a 15-fold increase of the XUV intensity. The spectral broadening from the quartz leads to strong half-cycle cutoffs, which implies that there are only two recollision events per pulse.

Rotating the polarization so that it is perpendicular to the $c$ axis of the quartz plate generates the second harmonic. The second harmonic suppresses every other half-cycle as it strongly increases the counts in the generated pulse (Fig. $3 \mathrm{~g}-\mathrm{i})^{24}$. The increase in harmonic amplitude between the two cases (Fig. 3f,i) implies that the second harmonic intensity is a few percent of the fundamental ${ }^{25,26}$. Changing the CEP by $\pi$ does not change the sign of the second harmonic because of the quartz crystal orientation. By scanning the CEP, we tune the recollision energy-and thus the photon energy - by more than an octave from 15 to $35 \mathrm{eV}$.

We can also generate attosecond pulses in krypton, as shown in Fig. $4 \mathrm{a}$, by simply increasing the driving laser-pulse energy. The generated spectrum is also tuneable from 20 to $45 \mathrm{eV}$ by only varying the CEP. In fact, the driving-pulse duration is so short that we completely turn off the generation for certain values of the CEP. We can further increase the generated photon energy to the soft X-ray spectral region by changing the generating medium to argon. Although the CEP-dependent amplitude modulation in the spectrum is not as strong as that in xenon and krypton, Fig. $4 \mathrm{~b}$ shows a single half-cycle cutoff that extends beyond $120 \mathrm{eV}$. The isolated attosecond pulse spectrum that we produce in argon is comparable to that typically produced with $800 \mathrm{~nm}$ pump pulses in neon ${ }^{27}$. However, with the well-known wavelength scaling of the gas-phase high-harmonic generation of $\lambda^{-5-6}$ (ref. 28), the comparative flux should be approximately $1 \%$. With the increased efficiency from the half-cycle pulse, we expect the generated XUV flux to be comparable. With higher pulse energies and with neon or helium as the generating medium, it will be possible to generate isolated attosecond pulses with photon energies that extend to the soft X-ray region ${ }^{29,30}$.

We now replace the gas jet as the generating medium with a quartz sample. With subcycle field control, we increase the damage-free intensity on the thin quartz (XUV generating) sample by approximately a factor of two ${ }^{31,32}$. This quartz sample is also $80 \mu \mathrm{m}$ thick, $\mathrm{X}$-cut with the laser polarization along the ordinary $(\Gamma-\mathrm{M})$ direction $^{33}$, with the same orientation as the quartz plate shown in Fig. 1. The CEP-dependent spectrogram is shown in Fig. 4c. Interestingly, the CEP dependence of the XUV generated in the quartz sample has a different character to that in the gas cases. Below $20 \mathrm{eV}$, we observe two bright regions at around 16 and $20 \mathrm{eV}$. The CEP value that maximizes the signal in 

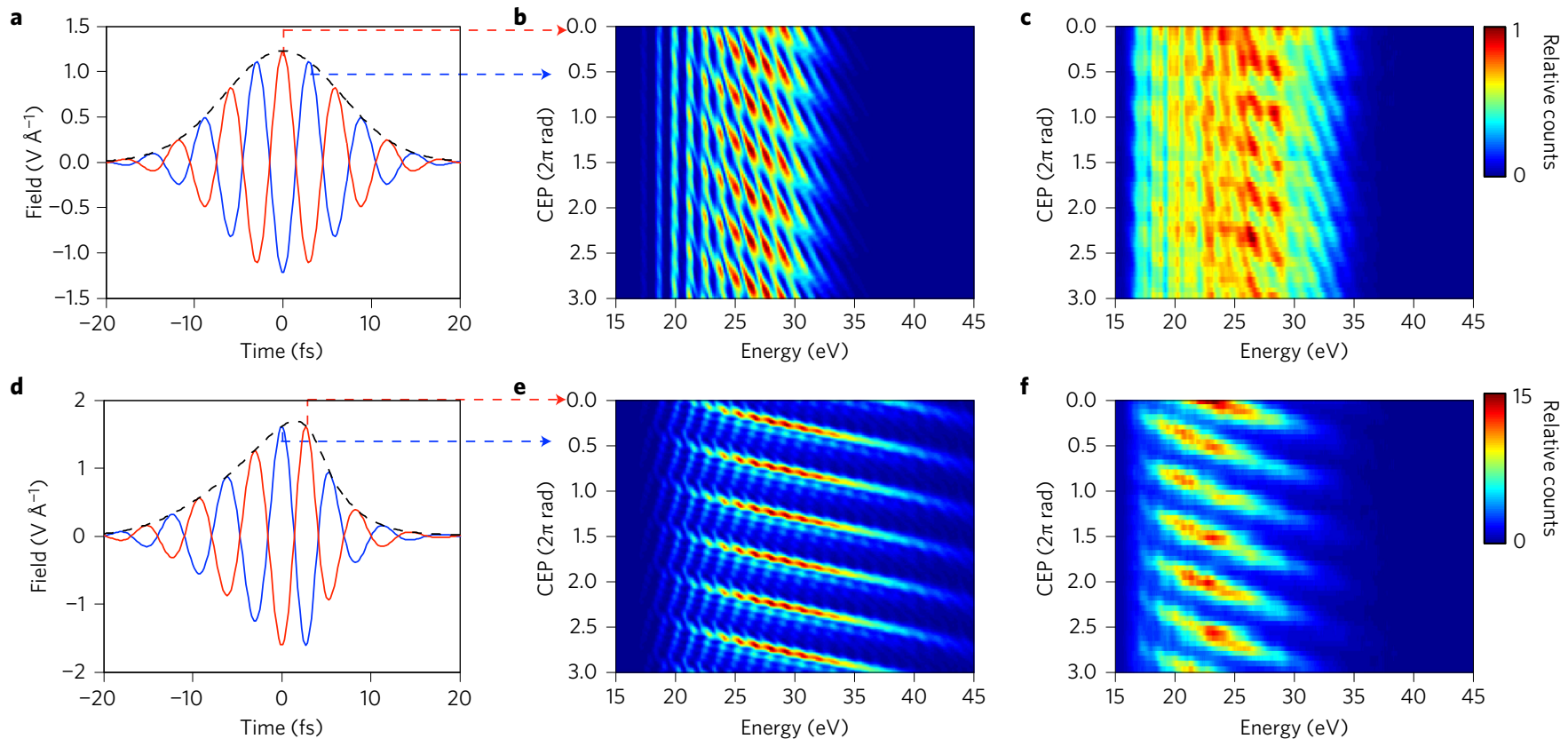

e $\rightarrow 0.0$

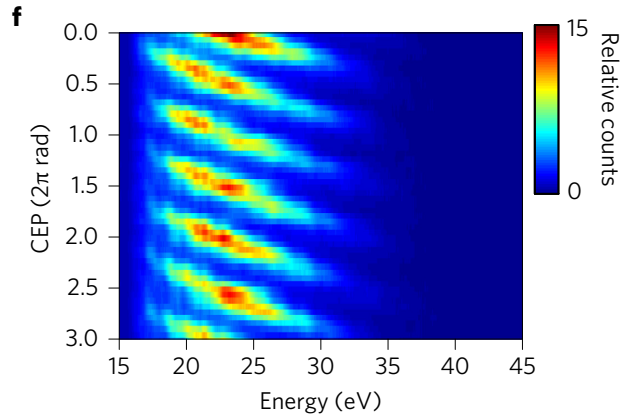

g

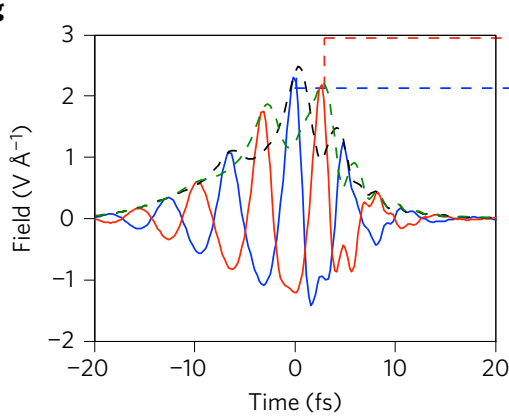

h
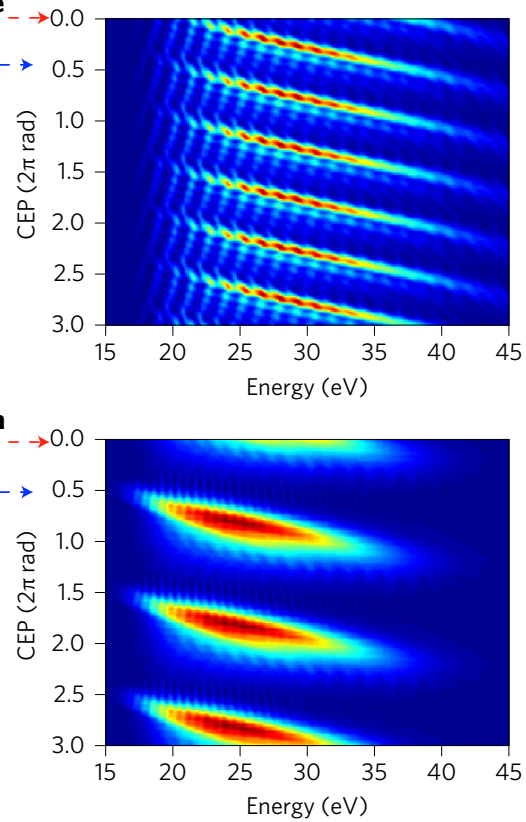

i

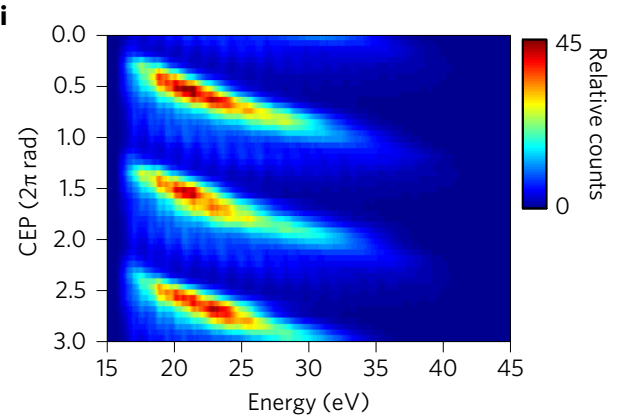

Figure 3 | CEP-dependent XUV spectra from xenon. a-c, Two-cycle pulse. $\mathbf{d}-\mathbf{f}$, After quartz polarization along the $c$ axis. $\mathbf{g}$-i, After quartz polarization perpendicular to the $c$ axis. The left column is the simulated field, the middle column is the simulated generated harmonics and the right column is the experiment. A two-cycle pulse has several recollision events, which lead to a discrete harmonic spectrum; the weak cutoff modulation implies the two half-cycle cutoffs are of comparable intensity. A pulse of single-cycle duration has two distinct recollision events. With the second harmonic present, every other ionization event is suppressed, which leads to an isolated attosecond pulse and a strong dependence of the ponderomotive energy on the CEP. Colour bars are the experimental amplitudes relative to $\mathbf{c}$.
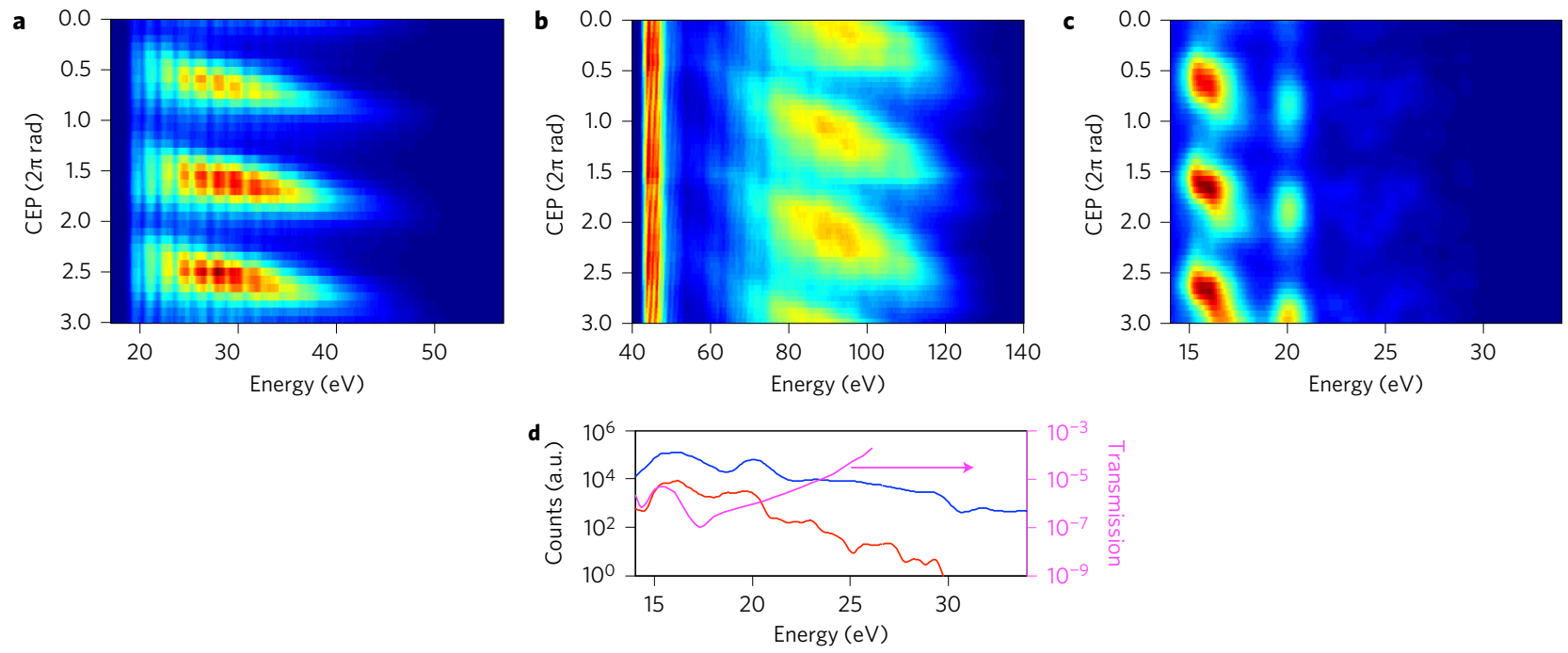

Figure 4 | XUV spectra from other media. a,b, We increase the intensity to extend the cutoff of krypton (a), showing strong modulation because of the CEP, and argon (b), where the isolated attosecond pulse extends from the Cooper minimum to the cutoff at $130 \mathrm{eV}$. c, CEP scan of the XUV radiation generated from quartz. d, The averaged spectrum over all the values of CEP in quartz for two-cycle pulses (red) and half-cycle pulses (blue) emulates the transmission through the final $100 \mathrm{~nm}$ (magenta). 

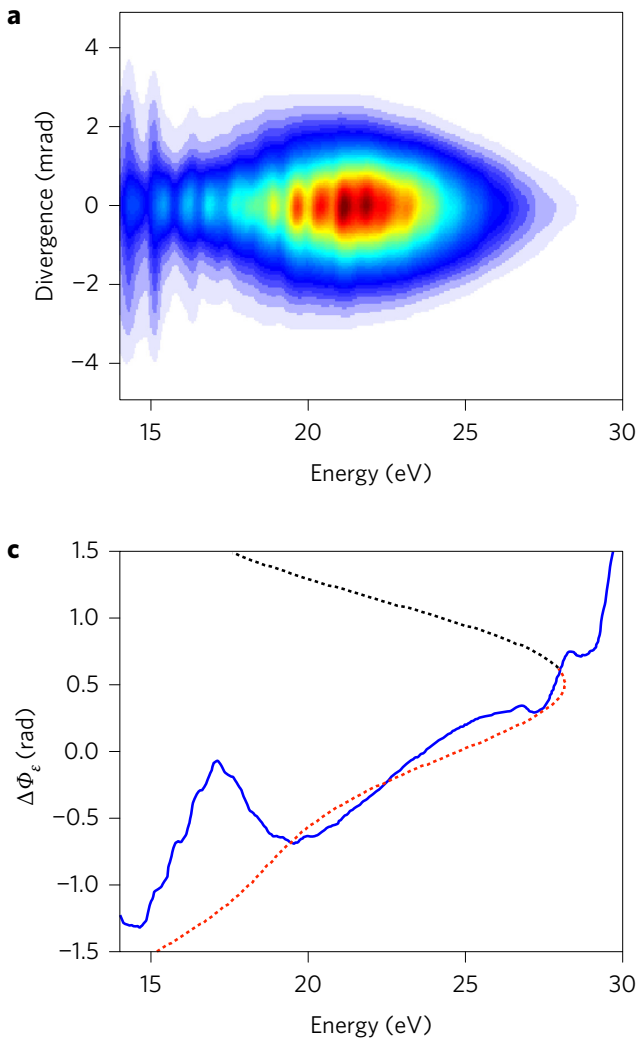

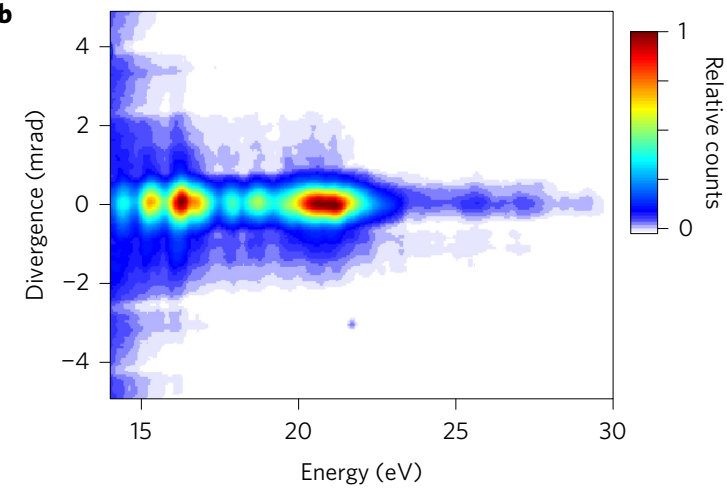

d

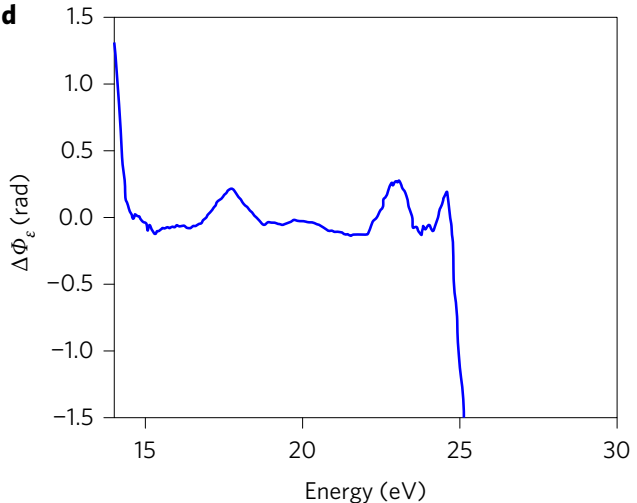

Figure 5 | Measured spectral intensity and phase of XUV radiation generated in xenon and quartz. a,b, We generate XUV radiation in xenon (a) and quartz (b) with comparable spectra (the magenta line is the extinction coefficient). $\mathbf{c}$, Reconstruction of the spectral emission phase, $\Delta \Phi_{\varepsilon}$, is in agreement with SFA calculations (dashed line, red for the short trajectory and black for the long trajectory). d, For the quartz sample, the spectral emission phase is flat.

the $16-20 \mathrm{eV}$ region behaves in a manner similar to that of gases. However, above $20 \mathrm{eV}$ there is a faint, but continuum, spectrum that extends to nearly $30 \mathrm{eV}$. Here the CEP that maximizes the signal is the same for all frequencies. The logarithm plot (Fig. 4d) is the CEP-averaged XUV spectra for a two-cycle (red) or halfcycle (blue) driving pulse, which shows the increased XUV flux caused, by over an order of magnitude, by the half-cycle pulse. We also plot the XUV transmission through the final $100 \mathrm{~nm}$ of quartz (magenta, ordinary ray) ${ }^{34}$, which shows the influence of the quartz transmission on the XUV spectrum.

We now investigate the XUV-emission time generated in xenon and quartz. To this end, we compare the XUV spatial and spectral modulation of the different media by in situ probing. Using a perturbative field with a relative intensity of $9 \times 10^{-4}$ of the fundamental and a relative angle of incidence of $10 \mathrm{mrad}$, we obtain the spectral emission phase $\mathrm{s}^{35}, \Delta \Phi_{\varepsilon} \propto \omega_{\mathrm{p}} \tau_{\epsilon}$, where $\omega_{\mathrm{p}}$ is the perturbing field frequency and $\tau_{\epsilon}$ is the emission time of the generated XUV radiation. The spatial-spectral profiles of the two media are shown in Fig. 5a,b. In the case of xenon, we see a continuum spectrum that spans $17-27 \mathrm{eV}$ with a relatively large divergence, which is well understood in the gas phase from the intensity-dependent high-harmonic wavefront ${ }^{36}$. There are also some narrow-band resonances around $15 \mathrm{eV}$ (refs 37,38). In the case of XUV from quartz (Fig. 5b), the spatial profile is less divergent than in the xenon case. We also observe that the XUV continuum is not uniform, but shows an amplitude dependence that is consistent with absorption-limited harmonic generation.

The reconstructed spectral emission phase is shown in Fig. 5c,d (the set-up and reconstruction of the emission phase is given in Supplementary Information.) The emission phase of the XUV generated in xenon (Fig. 5c) agrees well with the strong-field approximation (SFA) model ${ }^{39}$, which has been confirmed in both in situ ${ }^{35,40,41}$ and ex situ measurements ${ }^{4,42,43}$. The dashed line in Fig. $5 \mathrm{c}$ shows the calculated emission time (red is the short trajectory, whereas black is the long trajectory).

As demonstrated in Fig. 5d, the quartz-originated XUV spectral emission phase shows a different character than that of xenon: the emission phase is nearly independent of frequency, and the flat phase implies a different generating mechanism from gas. Additionally, the modulation of the XUV occurs at twice the probe oscillation frequency (Supplementary Information). The generation mechanism for high-harmonic radiation is different in quartz to that in xenon, and is consistent with an intraband transition model ${ }^{18,44}$.

\section{Conclusion}

For multicycle pulses, dielectrics and gases differ in many ways, but most prominently by how rapid avalanche ionization in solids limits the usable intensity. With subcycle transients, we are able to avoid avalanche ionization and the tolerance of dielectrics-like gasesfor intense light becomes typical of their ionization potential or bandgap. These transients allow dielectrics and gases to intermingle in experiments with little need for intervening optical elements. Thus, we can integrate solids and gases in attoscience and elsewhere. Solids, with their potential for engineering, offer important new opportunities. They can be patterned with interstitial layers in the beam-propagation direction to optimize pulse synthesis. They can also be patterned in the transverse direction to control the wavefront and to influence beam quality.

In summary, we use a few-cycle infrared source to benefit from the low dispersive properties of quartz. We use an unengineered solid (first quartz plate) to create subcycle field transients. In this manner, we generate attosecond pulses through the combination of the subcycle field transient and high-harmonic generation. With no intervening elements, we are able to tune optically the 
generated XUV energy by over an octave. Furthermore, we are able to increase significantly the XUV flux generated from a sample (second quartz plate) without damage, and characterize the attosecond pulse.

\section{Methods}

Methods and any associated references are available in the online version of the paper.

Received 9 May 2017; accepted 17 July 2017; published online 21 August 2017

\section{References}

1. Baltuska, A. et al. Attosecond control of electronic processes by intense light fields. Nature 421, 611-615 (2003).

2. Sansone, G. et al. Isolated single-cycle attosecond pulses. Science 314, 443-446 (2006)

3. Corkum, P. B. \& Krausz, F. Attosecond science. Nat. Phys. 3, 381-387 (2007).

4. Goulielmakis, E. et al. Single-cycle nonlinear optics. Science 320, 1614-1617 (2008).

5. Krausz, F. \& Ivanov, M. Attosecond physics. Rev. Mod. Phys. 81, 163-234 (2009).

6. Ghimire, S. et al. Observation of high-order harmonic generation in a bulk crystal. Nat. Phys. 7, 138-141 (2011).

7. Vampa, G. et al. Linking high harmonics from gases and solids. Nature $\mathbf{5 2 2}$, 462-464 (2015).

8. Krauss, G. et al. Synthesis of a single cycle of light with compact erbium-doped fibre technology. Nat. Photon. 4, 33-36 (2010).

9. Huang, S. W. et al. High-energy pulse synthesis with sub-cycle waveform control for strong-field physics. Nat. Photon. 5, 475-479 (2011).

10. Wirth, A. et al. Synthesized light transients. Science 334, 195-200 (2011).

11. Cox, J. A., Putnam, W. P., Sell, A., Leitenstorfer, A. \& Kaertner, F. X. Pulse synthesis in the single-cycle regime from independent mode-locked lasers using attosecond-precision feedback. Opt. Lett. 37, 3579-3581 (2012).

12. Chia, S.-H. et al. Two-octave-spanning dispersion-controlled precision optics for sub-optical-cycle waveform synthesizers. Optica 1, 315-322 (2014).

13. Fattahi, H. Sub-cycle light transients for attosecond, X-ray, four-dimensional imaging. Contemp. Phys. 57, 580-595 (2016).

14. Hassan, M. Th. et al. Optical attosecond pulses and tracking the nonlinear response of bound electrons. Nature 530, 66-70 (2016).

15. Lu, C.-H. et al. Generation of intense supercontinuum in condensed media. Optica 1, 400-406 (2014).

16. He, P. et al. High-efficiency supercontinuum generation in solid thin plates at 0.1 TW level. Opt. Lett. 42, 474-477 (2017).

17. Franken, P. A., Hill, A. E., Peters, C. W. \& Weinreich, G. Generation of optical harmonics. Phys. Rev. Lett. 7, 118-119 (1961).

18. Garg, M. et al. Multi-petahertz electronic metrology. Nature 538, 359-363 (2016)

19. Husakou, A. V. \& Hermann, J. Supercontinuum generation of higher-order solitons by fission in photonic crystal fibers. Phys. Rev. Lett. 87, 203901 (2001).

20. Gaeta, A. L. Catastrophic collapse of ultrashort pulses. Phys. Rev. Lett. 84, 3582-3585 (2000).

21. Wagner, N. L. et al. Self-compression of ultrashort pulses through ionizationinduced spatio-temporal reshaping. Phys. Rev. Lett. 93, 173902 (2004).

22. Kim, K. T. et al. Petahertz optical oscilloscope. Nat. Photon. 7, 958-962 (2013).

23. Corkum, P. B. Plasma perspective on strong-field multiphoton ionization. Phys. Rev. Lett. 71, 1994-1997 (1993).

24. Mashiko, H. et al. Double optical gating of high-order harmonic generation with carrier-envelope phase stabilized lasers. Phys. Rev. Lett. 100, 103906 (2008).

25. Fieß, M. et al. Attosecond control of tunneling ionization and electron trajectories. New J. Phys. 13, 033031 (2011).

26. Hammond, T. J., Kim, K. T., Zhang, C., Villeneuve, D. M. \& Corkum, P. B. Controlling attosecond angular streaking with second harmonic radiation. Opt. Lett. 40, 1768-1770 (2015).

27. Zhao, K. et al. Tailoring a 67 attosecond pulse through advantageous phasemismatch. Opt. Lett. 37, 3891-3893 (2012).

28. Shiner, A. D. et al. Wavelength scaling of high harmonic generation efficiency. Phys. Rev. Lett. 103, 073902 (2009).
29. Silva, F., Teichmann, S. M., Cousin, S. L., Hemmer, M. \& Biegert, J. Spatiotemporal isolation of attosecond soft X-ray pulses in the water window. Nat. Comm. 6, 6611 (2015).

30. Teichmann, S. M., Silva, F., Cousin, S. L., Hemmer, M. \& Biegert, J. 0.5-keV soft X-ray attosecond continua. Nat. Comm. 7, 11493 (2016).

31. Stuart, B. C., Feit, M. D., Rubenchik, A. M., Shore, B. W. \& Perry, M. D. Laserinduced damage in dielectrics with nanosecond to subpicosecond pulses. Phys. Rev. Lett. 74, 2248-2251 (1995).

32. Carr, C. W., Radousky, H. B. \& Demos, S. G. Wavelength dependence of laserinduced damage: determining the damage initiation mechanisms. Phys. Rev. Lett. 91, 127402 (2003).

33. Luu, T. T. et al. Extreme ultraviolet high-harmonic spectroscopy of solids. Nature 521, 498-502 (2015)

34. Palik, E. D., Ghosh, G. \& Prucha, E. J. Handbook of Optical Constants in Solids (Academic, 1998).

35. Kim, K. T. et al. Manipulation of quantum paths for space-time characterization of attosecond pulses. Nat. Phys. 9, 159-163 (2013).

36. Frumker, E., Paulus, G. G., Niikura, H., Villeneuve, D. M. \& Corkum, P. B. Frequency-resolved high-harmonic wavefront characterization. Opt. Lett. 34, 3026-3028 (2009)

37. Rothhardt, J. et al. Enhancing the macroscopic yield of narrow-band high-order harmonic generation by Fano resonances. Phys. Rev. Lett. 112, 233002 (2014).

38. Bengtsson, S. et al. Space-time control of free induction decay in the extreme ultraviolet. Nat. Photon. 11, 252-258 (2017).

39. Lewenstein, M., Balcou, P., Ivanov, M. Y., L'Huillier, A. \& Corkum, P. B. Theory of high-harmonic generation from low frequency laser fields. Phys. Rev. A 49, 2117-2132 (1994)

40. Dudovich, N. et al. Measuring and controlling the birth of attosecond XUV pulses. Nat. Phys. 2, 781-786 (2006).

41. Zhang, C., Brown, G. G., Kim, K. T., Villeneuve, D. M. \& Corkum, P. B. Full characterization of an attosecond pulse generated using an infrared driver. Sci. Rep. 6, 26771 (2016).

42. Mairesse, Y. et al. Attosecond synchronization of high-harmonic soft X-rays. Science 302, 1540-1543 (2003).

43. Hammond, T. J., Brown, G. G., Kim, K. T., Villeneuve, D. M. \& Corkum, P. B. Attosecond pulses measured from the attosecond lighthouse. Nat. Photon. 10, 171-175 (2016).

44. Wu, M., Ghimire, S., Reis, D. A., Schafer, K. J. \& Gaarde, M. B. High-harmonic generation from Bloch electrons in solids. Phys. Rev. A 91, 043839 (2015).

\section{Acknowledgements}

We thank K. Oudatchin for providing the X-ray diffraction data used to verify the X-cut crystal orientation. We also thank T. Brabec and C. Macdonald for useful discussions, and D. Crane and B. Avery for technical assistance. This material is based on work supported by the Air Force Office of Scientific Research (AFOSR) under award number FA9550-16-10109, the AFOSR Multidisciplinary University Research Initiative grant number FA955015-1-0037 and US Army Research Office grant W911NF-14-1-0383. The authors also acknowledge financial support from the National Research Council of Canada, the Natural Sciences and Engineering Research Council of Canada and the Canadian Foundation for Innovation and the Ontario Research Fund.

\section{Author contributions}

T.J.H. and S.M. conceived the initial idea. T.J.H., S.M. and C.Z. performed the experiment T.J.H. and P.B.C. provided the model. T.J.H. provided the theory and analysed the experimental data. G.V. and D.K. provided the model for the quartz. T.J.H. and P.B.C prepared the initial manuscript. All the authors contributed to the preparation of the manuscript.

\section{Additional information}

Supplementary information is available in the online version of the paper. Reprints and permissions information is available online at www.nature.com/reprints. Publisher's note: Springer Nature remains neutral with regard to jurisdictional claims in published maps and institutional affiliations. Correspondence and requests for materials should be addressed to T.J.H.

\section{Competing financial interests}

The authors declare no competing financial interests. 


\section{Methods}

We pump an optical parametric amplifier (Light Conversion HE-TOPAS) to generate a signal and idler at 1.4 and $1.8 \mu \mathrm{m}$, respectively. The idler is CEP stabilized, and initially has a $70 \mathrm{fs}$ pulse duration and $1 \mathrm{~mJ}$ of energy. It is sent into a hollow core fibre $(500 \mu \mathrm{m}$ diameter) filled with 1 bar argon and differentially pumped to create a spectrum that supports two-cycle pulses at $1.8 \mu \mathrm{m}$. The output pulse is compressed using $3 \mathrm{~mm}$ of fused silica. For the harmonic-generation experiments, up to $450 \mu \mathrm{J}$ is delivered into the vacuum chamber. The focussing optics are two $1 \mathrm{~m}$ silver mirrors, separated by $70 \mathrm{~cm}$ to focus the beam down to $165 \mu \mathrm{m}$ for a maximum intensity of $5 \times 10^{13} \mathrm{~W} \mathrm{~cm}^{-2}$. We control the intensity by changing the beam size with an iris, with typical working conditions using a minimum waist of $180 \mu \mathrm{m}$ and a peak intensity of $2 \times 10^{13} \mathrm{~W} \mathrm{~cm}^{-2}$. We scan the CEP via a pair of thin fused-silica wedges. Further details for the experimental set-up are given in Supplementary Information.

Data availability. The data that support the plots within this paper and other findings of this study are available from the corresponding author on reasonable request. 